\title{
Bilingualism and bimodal code-blending among deaf ASL-English bilinguals
}

\author{
Marjorie Herbert \& Acrisio Pires*
}

\begin{abstract}
The audiologically deaf members of the American Deaf community display bilingual competence in American Sign Language (ASL) and English, although their language acquisition trajectories often involve delayed exposure to one or both languages. There is a great deal of variation in terms of production among these signers, ranging from very ASL-typical to productions that seem to display heavy English influence. The latter, mixed productions, coined "Contact Signing" by Lucas \& Valli (1992), could be representative of a type of codeswitching, referred to as 'code-blending' in sign language-spoken language contexts (e.g. Baker \& Van den Bogaerde 2008), in which bilinguals invoke knowledge of their two grammars in concert, or these productions could be more like a mixed language, in which a third grammar, distinct from both ASL and English, constrains them. We argue, based on the analysis of our corpus of naturalistic data collected in an all-deaf sociolinguistic environment, that Contact Signing provides evidence for code-blending, given the distribution of English vs. ASL-based language properties in the production data from the participants in our study.
\end{abstract}

Keywords. American Sign Language, code-blending, sign language-spoken language contact, bilingualism, deaf studies

1. Introduction. This study examines effects of bilingualism and language contact between ASL and English, specifically within the audiologically deaf ${ }^{1}$ individual members of the American Deaf community. The arguably mixed productions of these signers are typically referred to as Pidgin Sign English (PSE). We adopt the more theoretically-neutral term for this particular language contact outcome, 'Contact Signing', or what we will refer to in this paper as CSign, as coined by Lucas \& Valli (1989; 1992). Those authors define CSign as "something that cannot be strictly described as ASL or as a signed representation of English" (1992: 18). In other words, the CSign productions of an individual have elements of natural ASL and elements of English, but they cannot be reduced to his individual mental grammar of natural ASL or of English. We adopt a hypothesis that the grammars or idiolects resulting from a language contact situation must be explainable at the individual, cognitive level in terms of a combination of linguistic and third factor principles (Chomsky 2005). The structure of this paper is as follows. This section outlines the acquisition trajectories and outcomes that are typical among the deaf members of the American Deaf community. Section 2 describes some language properties that have been proposed as features of CSign. Section 3 reviews previous descriptions and analyses of CSign. The remaining sections present the methodology and results of the current study.

1.1. THE GENETICS OF DEAFNESS AND DEAF LANGUAGE ACQUISITION. The population of interest for this study is the subset of the Deaf community that is deaf. Hearing, as a human biological capacity, encompasses a spectrum of individual differences, rather than a binary

\footnotetext{
*Thank you to Jose Galofre, Anne Spence, Sarah Bonello, Morgan Durrow, Hillari Varshaw, and Mason Friend for all their hard work helping annotate our corpus, and to Marlyse Baptista and Jonathan Brennan for their invaluable critiques and suggestions. Authors: Marjorie Herbert, University of Michigan (mgherb@umich.edu) \& Acrisio Pires, University of Michigan (pires@umich.edu).

${ }^{1}$ Here we use the notational convention of 'capital D' Deaf to refer to the Deaf community/Deaf culture versus deaf with a lower-case 'd' to refer to audiological status.
} 
opposition such as 'deaf/hearing' or even 'deaf/hard-of-hearing', therefore we have included in this study hard-of-hearing individuals, deaf individuals with Cochlear Implants, and individuals who were born hearing and became deaf in early childhood. The broad aim of the project is to characterize the mental grammars underlying the sign language production of ASL-English bilinguals whose access to the spoken language was limited physiologically to a large (albeit variable) extent during acquisition.

Unlike other minority languages in the U.S., ASL is not typically transmitted from one generation to the next. Because of the genetics of deafness, the majority of deaf children (9095\%) are born to hearing parents, whereas only about 5-10\% acquire ASL natively from Deaf parents. Most of the remaining members of the Deaf community learn ASL when they enter school. Some learn from Deaf teachers, the privileged few of their peers born to Deaf parents, and/or from hearing educators fluent in ASL (Lillo-Martin 1999). Many others, however, acquire their manual languages from deaf and hearing members of their school community who rely on Manually Coded English (MCE) systems, or signed communication systems invented for use in deaf education to represent English visually.

This brings up another defining characteristic of the Deaf community: bilingualism. Unlike other instances of stable bilingualism in the world, in which many individuals might not have knowledge of both languages in contact in their regions, bilingualism at the individual level is the norm rather than the exception in the Deaf community (Lucas \& Valli 1992). Virtually all American Deaf who have some education will have some knowledge of English, although the levels of that knowledge vary widely across individuals, due primarily to the constraints that have been placed on their early language acquisition.

Finally, there are a few sociolinguistic variables that have been found to be significant in the American Deaf community that are not found in the spoken language majority, such as family history (as in deaf or hearing, signing or not-signing), education type (i.e. mainstream public school with hearing students versus a school for the Deaf with a majority-deaf peers), and specific school for the Deaf attended; see also Lucas (2001). Lee (1982) also notes qualitative differences in the signed productions of hearing as opposed to deaf signers, so audiological status plays a significant role as well. These factors have also been considered in the current study.

2.The linguistic features of contact signing. Table 1 shows some features of CSign, as proposed by Lucas \& Valli (1992), in contrast with their hypothesized grammatical counter-parts in natural ASL. One pattern that emerges from this table is that CSign often incorporates elements from English in different ways, but it also maintains some natural ASL structures. CSign also tends to transform English grammatical elements for the manual modality, and these functional items often form structures whose parallels in natural ASL have quite a different form.

The second row of Table 1 illustrates this phenomenon. Natural ASL typically encodes spatial relationships among agents and objects with classifier constructions, in cases in which English uses prepositional phrases, as illustrated in example (1). As in (1a), a signer would typically articulate the arguments of the classifier, CAT and TABLE in this case, and would then articulate the classifier by using the handshape for animal entities in order to form an arced path through space, starting at the location of the cat and ending at the location of the table. ${ }^{2}$ By

\footnotetext{
${ }^{2}$ Sign languages classifiers are handshapes that can be used to represent a noun in the signing space. Classifier constructions tend to represent the most visually salient characteristics of their referents, like flatness or roundness,
} 
contrast, (1b) makes use of the ASL lexical verb JUMP, rather than a classifier, and adds the MCE preposition ON.

\begin{tabular}{|c|c|}
\hline Natural ASL & CSign \\
\hline $\begin{array}{l}\text { WHY or REASON + non-manuals; } \\
\text { ASL use of space + ASL indices }{ }^{3}\end{array}$ & $\begin{array}{l}\text { BECAUSE; } \\
\text { constructions with THAT (ex. relative } \\
\text { clause), MCE conjunctions, comparative } \\
\text { phrases (ex. MORE + THAN) }\end{array}$ \\
\hline $\begin{array}{l}\text { Classifier Constructions; } \\
\text { ASL verbs, constructions }\end{array}$ & $\begin{array}{l}\text { MCE prepositions; } \\
\text { English-like constructions with MCE } \\
\text { prepositions/verbal particles (ex. } \\
\text { DEPEND ON, GIVE MEANING TO) }\end{array}$ \\
\hline $\begin{array}{l}\text { English mouthing lexicalized to } \\
\text { individual ASL signs }\end{array}$ & $\begin{array}{l}\text { English mouthing produced continuously } \\
\text { and/or spread across a signed utterance }\end{array}$ \\
\hline ASL inflectional and derivational morphology & $\begin{array}{l}\text { Some fingerspelled forms representing } \\
\text { English affixes (ex. \#ING, \#MENT }{ }^{4} \text { ), } \\
\text { Some reduced ASL morphology }\end{array}$ \\
\hline
\end{tabular}

(1) a. CAT TABLE CL: animal jumps up

b. CAT JUMP ON TABLE.

'The cat jumps on the table.'

The third row of the table discusses the status of mouthing ${ }^{5}$ in ASL and CSign. Sandler \& Lillo-Martin (2006) argue that only a small percentage of signs across sign languages are accompanied by spoken language mouthing. Their idea is that, although lexicalized mouthing can be characteristic of natural ASL production, it is restricted to only some individual ASL signs. They make the point in a footnote that this conclusion is based on mouthing that accompanies productions in "real sign languages", not "contrived systems" like Signed English (2006: 104). However, Lucas \& Valli (1992) make the argument that CSign cannot be reduced to the grammar of natural ASL, neither can it be characterized as Signed English (a form of MCE), which is an invented and often contrived system, or any other form of unnatural linguistic production. The results from our study show that continuous, sometimes 'stretched' mouthing (a mouthed word that stretches over multiple, contiguous signs) does occur often in CSign. Furthermore, individual ASL signs are not always accompanied by the same mouthing in all contexts in CSign, which would be expected if they were lexicalized mouthing: for example, the sign FINISH, which has been argued to be accompanied by lexicalized mouthing, sometimes cooccurs in our data with the mouthing finish, whereas, in other contexts, it co-occurs with already. We will argue in section 6 that this CSign production is determined by at least two

orientation in space, and typical movements or actions of that object (Hong 2008). Figure 1 below shows an ASL classifier (Sandler \& Lillo-Martin 2006).

${ }^{3}$ ASL indices are sometimes referred to as ASL pronouns and determiners. In ASL, referents can be associated with spatial locations (Liddell 1990a). Non-present referents are assigned an arbitrary location in space, which, moving forward in the discourse, indexes that referent. The signer points to that location in space to mention that referent.

${ }^{4}$ We adopt the convention established in the literature of adding a pound sign, '\#', in front of fingerspelled items that have been proposed in previous work as lexicalized fingerspellings in ASL. However, we take no theoretical stance in this paper as to their status within the natural ASL lexicon.

${ }^{5}$ English mouthing is the silent oral articulation of English words to accompany signers' manual productions. 
grammars, one of which shows properties of a natural sign language.

By contrast, a more typical strategy in ASL is to resort to fingerspelling ${ }^{6}$ an English word, when the semantically related sign in ASL does not quite match the meaning of that English word. Example 4, also from Lucas \& Valli (1992, appendix 2), illustrates this point.

\section{HAVE A DEAF PRESIDENT ALSO ROLE \#MODEL WHEN MAKE POINT WORTH\#WHILE "WELL"}

'To have a deaf president and also a deaf role model, when he makes a point, it will be worthwhile.'

This signer fingerspells the English word 'model', indicated in 4 with '\#' (the standard sign for MODEL in ASL typically refers to a person who works in the fashion industry as a model). Similarly, there is not a perfect translation equivalent in ASL to the English word 'worthwhile', so, interestingly, this signer has produced a sign+fingerspelling combination.

According to Lucas \& Valli (1992), examples such as (1b) and (2) are representative of CSign, as summarized in Table 1. Similarly, considering the next row of the table, sometimes CSign can recruit ASL derivational and inflectional morphology, but at other times, it uses fingerspelled representations of the affixes and suffixes in English that have a corresponding function before or after the ASL sign for the morphological stem.

3. Previous Analyses of Contact Signing. The prevailing view before Lucas \& Valli (1989; 1992), which persists in much of the literature to this day, is that CSign is a pidgin language system, and it arose to facilitate communication between adult deaf signers and less skilled hearing signers. Woodward et al. (1973) coined CSign's more common appellation, Pidgin Sign English (PSE), and characterized it as a pidgin system, since they claimed reduced morphology and grammatical marking, similar to the supposed grammatical reduction of pidgins developing in spoken language contact settings, as the hallmark of PSE in the American Deaf community. Although he also assumes PSE arose from deaf-hearing interactions, Cokely (1983), on the other hand, argues the conditions for pidgin formation could not possibly be met in this particular community and characterizes it as a type of foreigner talk.

Lucas \& Valli $(1989 ; 1992)$ set out to resolve the debate, controlling more stringently for the types of interactions being observed (deaf/hearing, L2-learner/L1-talker) than previous studies had and building into their procedure an all-deaf condition to contrast with a condition with a hearing interviewer. They found CSign arises in all of the sociolinguistic situations they investigated and that the language background of the interlocutors could not fully account for this result. Furthermore, more CSign was not found in deaf-hearing interactions versus deaf-only situations, nor the reverse for that matter.

3.1. A LingUiSTIC CONTINUUM. Stokoe (1969) first characterized the ASL-English contact situation in the U.S. as one of stable diglossia, with ASL as the low (L) variety and spoken English/more English-like forms of signing as the high (H). Lee (1982), building off of Stokoe, Cokely, and other authors who understand CSign in terms of a diglossic continuum, argues there are two separate, qualitatively different continua, one for hearing signers (PSEh)

\footnotetext{
${ }^{6}$ In fingerspelling each letter of the English alphabet is assigned a hand configuration (handshape + hand orientation in space); signers rapidly transition among these configurations to spell out English orthographic words. It can be a special type of lexical borrowing from English into ASL, since it involves a very indirect representation of English phonology, i.e. through English orthography (Battison 1978; Davis 1989; Keane et al. 2012).
} 
and one for deaf signers (PSEd; Lee 1982: 131). In section 6 we will propose a bilingual approach to the signed production of deaf/hard-of-hearing individuals.

3.2. A SEPARATE LANGUAGE VARIETY. Based on results from their 1992 study, Lucas \& Valli conclude instead that CSign is a "third system", meaning it results from contact between two languages, but "must be analyzed in terms of its own structure rather than in terms of one language or the other" (p. 147). They make two main arguments to conclude that CSign must represent a 'third system' phenomenon: i) in CSign, features of ASL and English are "hopelessly mixed" (p. 100), and ii) mutual intelligibility across participants was never a problem in their study. Berent (2004), while acknowledging the huge significance of their study, points out flaws in each argument. First, the fact that features from both English and ASL show up in CSign is hardly surprising; that would be expected in any contact situation. The second piece of evidence that Lucas \& Valli invoke is not a reliable indicator of a third grammar constraining that system, since all of their participants were fluent in both ASL and spoken English.

3.3. CODE-BLENDING. Emmorey et al coin the term "code-blending" to refer to the type of codeswitching that is particularly common in bimodal bilinguals: simultaneous realization (2005). They define code-blends as any production in which "ASL signs are produced simultaneously with [spoken] English words" (p. 666). Baker and Van den Bogaerde (2008) refer to the mixed productions elicited from deaf and hearing infants acquiring Dutch Sign Language (NGT), both those that involve fully voiced Dutch and mouthed spoken Dutch elements (mouthing), as code-blending. For the purposes of this study, we will refer to the analysis that deaf CSign productions are constrained by their grammars of ASL and English in concert as code-blending, adopting Baker and Van den Bogaerde's perspective, since this term captures the similarity that code-blending has to spoken language code-switching.

\section{The Present Study}

4.1. PARTICIPANTS. The target population for this study is deaf adults who communicate regularly in ASL and have some knowledge of English. Adult participants (age 18 or older) were selected for the study if they self-reported in a preliminary language background survey that they began acquiring ASL before age 7 and that they use spoken or written English on at least a weekly basis. All subjects were asked to bring with them for the study a friend they feel comfortable signing with, in order to ensure they were comfortable and engaged in "bilingual mode" (Grosjean 1984). Each friend participant was also tested in the study, but their language background was recorded only in the course of the study. Fifteen pairs and three triads of participants were tested in total $(n=39)$, but for the purposes of the analysis reported in this paper, we focus on ten pairs $(n=20 \text {, age range } 18-65 \text {, mean age } 41)^{7}$, randomly selected among the participants. Eighteen participants were white and two were Pacific Islanders. Every participant reported having received a high school diploma, and of the sixteen participants who were past typical college age (25+), twelve of these had a bachelor's degree or higher. Ten of the twenty reported the onset of their ASL exposure as before age 7, seven of whom were native signers (ASL exposure from birth). The remaining ten participants (the friend participants) reported their age of ASL onset as in their teens, a relatively common outcome for deaf individuals even today. Interestingly, all participants, regardless of their initial age of exposure, reported a preference for manual communication over English. In terms of audiological status, five were hard-of-hearing,

${ }^{7}$ The final group included in this paper was actually a triad $(n=3)$, not a pair - one member of this group was a hearing child of deaf adults (CODA) who was excluded from the analysis, since we only considered individuals with reported hearing loss. 
and the remaining fifteen were deaf. Six attended a school for the Deaf, whereas fourteen went to a mainstream, public school.

4.2. Methods. All participants interacted exclusively with a congenitally deaf confederate and their ASL-signing friend for the duration of their participation in the study. The confederate carried out all the interaction with the participants, using sign language.

4.3. PROCEDURE. The study included three main tasks:

(i) Participant pairs were interviewed during the first 15-20 minutes of the study (condition 1). These interview questions, sourced from Lucas \& Valli's (1992), concerned issues relevant to the Deaf community and were designed to elicit more ASL-like signing.

(ii) The confederate left the room after asking the two signers to continue their interaction for the next 15-20 minutes of the study (condition 2). In this task, participants were simply asked to continue the conversation that arose naturally from the interview questions.

(iii) Third, each subject individually was shown two black-and-white paper cartoons without language (condition 3 ). S/he was given a few minutes to study each cartoon, and then asked to recount the sequence of events to the confederate, which lasted less than five minutes.

4.4. DATA ANALYSIS. For conditions 1 and 2, the interview and conversation conditions, fiveminute representative clips were extracted from the midpoint in time for each. These clips, along with the entirety of the story-telling condition, condition 3, were coded with the language transcription software ELAN and analyzed for this study.

We analyzed six linguistic features of CSign, as identified by Lucas \& Valli: English mouthing, fingerspelling and English-based prepositions and conjunctions were hypothesized to be English-related properties, while ASL classifiers, ASL indices, ${ }^{8}$ and ASL agreeing verbs ${ }^{9}$ were treated as ASL-related. We hypothesized that the ASL-related properties would positively predict one another and negatively predict the English-related properties, and vice-versa (see section 5 for how the predictions were tested). All of these properties except for English mouthing are produced manually, so they were coded at the sign level. English mouthing is produced orally and normally simultaneously with signs, so it was coded on a distinct level. Properties that were selected must be strongly motivated, either by Lucas \& Valli 1992 or the broader linguistics literature on ASL, as a property of ASL-like signing or of more English-like signing.

5. Results. We take two linguistic properties, one argued to relate to natural ASL, and the other one argued to relate to spoken English, as the outcomes that other hypothesized properties of would CSign predict. The ASL-related property is classifier usage, as classifiers seem to be an irrefutably integral part of the natural ASL grammar/lexicon (Brentari \& Padden 2001). English mouthing is the English-related outcome; one of the most robust findings of Lucas \& Valli's (1992) study is that sustained mouthing across signed discourses is indicative of CSign. We used generalized linear mixed models (GLMM) in the statistics package R to analyze the data.

\footnotetext{
${ }^{8}$ Some researchers hypothesize that these indices are akin to determiners and pronouns in spoken language, but this view is not accepted unilaterally (see Liddell 2000). The more neutral term 'index' is adopted in this paper.

${ }^{9}$ Agreeing verbs agree spatially with their argument(s), which typically means the motion of the verb will originate at a location in space that refers to the subject and terminate at a location that refers to the object (Padden 1987).
} 


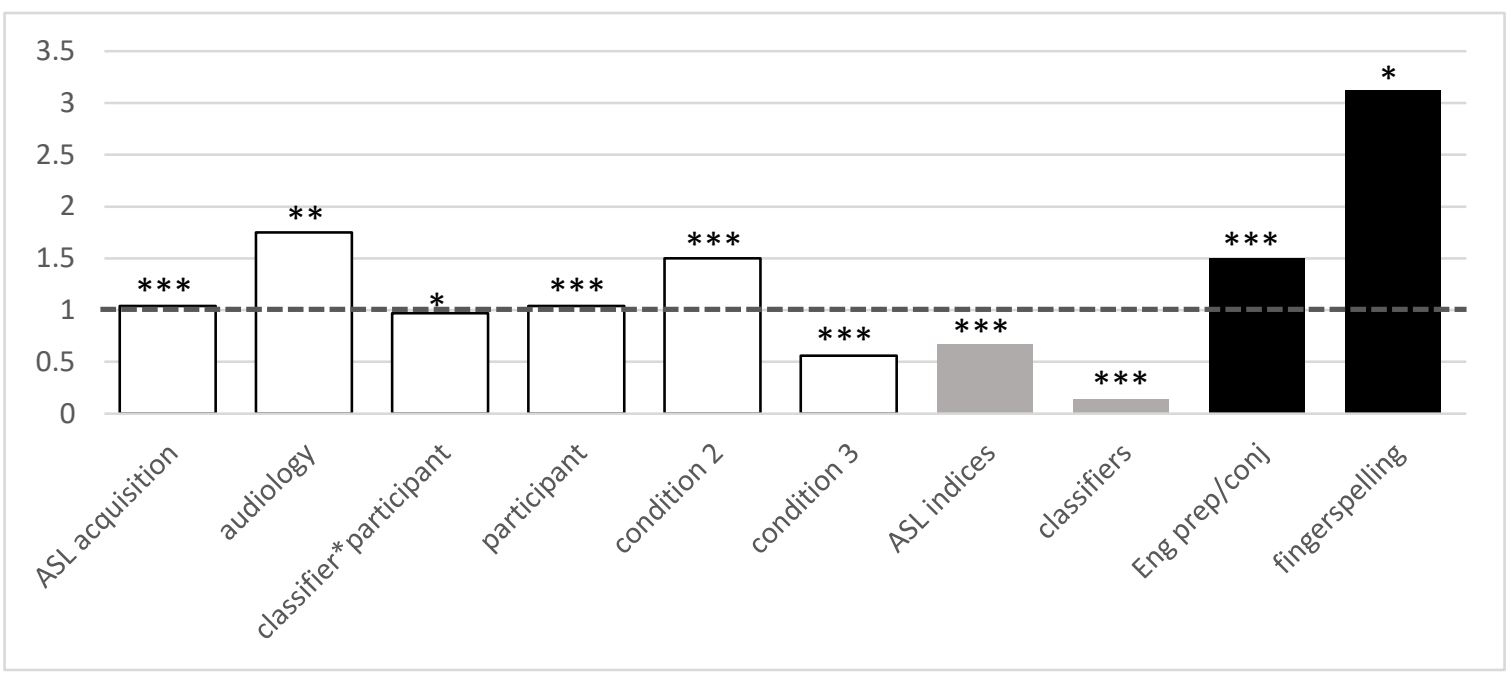

Figure 2. The odds of English mouthing co-occurring with each predictor.

5.1. ENGLISH MOUTHING AS OUTCOME. In the first GLMM, English mouthing was taken as the outcome of several predictors ${ }^{10}$. We conducted an incremental analysis, incorporating first the five other language properties that were coded, then the demographic variables we constructed from the language background questionnaire each participant completed, such as age of exposure to ASL, type of school attended, etc. Figure 2 represent the odds for the variables that were significant predictors of English mouthing (the figures only show significant predictors). The other English-indexed properties, fingerspelling (fs) and English prepositions and conjunctions, positively predicted mouthing, as expected, with odds of 3.12 and 1.50 respectively, whereas two of the ASL-indexed properties, classifiers and ASL indices, negatively predicted mouthing, with odds of 0.14 and 0.67 , respectively, also as expected. ASL verbal agreement, as an ASL-indexed property, was expected to be a negative predictor; however, this variable was not found to be a statistically significant predictor of English mouthing. Regarding demographic information, participants who identified their audiological status (audiology in Fig. 2) as hard-of-hearing were 1.75 more likely to mouth across conditions, as compared to their deaf counterparts who reported either severely degraded or no previous or current auditory English input. There were three continuous variables found to be significant positive predictors of English mouthing. First, as the age participants reported as the onset of their ASL exposure increased (ASL acquisition in Fig. 2), rates of English mouthing also increased (number of signs accompanied by mouthing/total number of signs produced by participant). Second, the participant variable as a significant predictor indicates that English mouthing is highly variable across participants. Third, analysis of the interaction between classifier and participant (classifier*participant) revealed that participants who used classifiers more often than average also produced English mouthing less than the average, once classifier usage (number of classifiers/total number of signs produced by that participant) was plotted against mouthing.

\footnotetext{
${ }^{10}$ The dotted line in Figure 2 represents odds=1.0, or at chance. Predictors with bars above 1.0 are positive predictors (a participant who produces a predictor at a higher than average frequency is more likely to produce English mouthing at an above average rate); predictors with bars below 1.0 are negative predictors of mouthing. The asterisks above each bar indicate that predictor's statistical significance: $\mathrm{p}=0,{ }^{\prime}{ }^{* * *}, ; \mathrm{p}=0.001,{ }^{* *}$ '; $\mathrm{p}=0.01,{ }^{\prime *}$ '.
} 


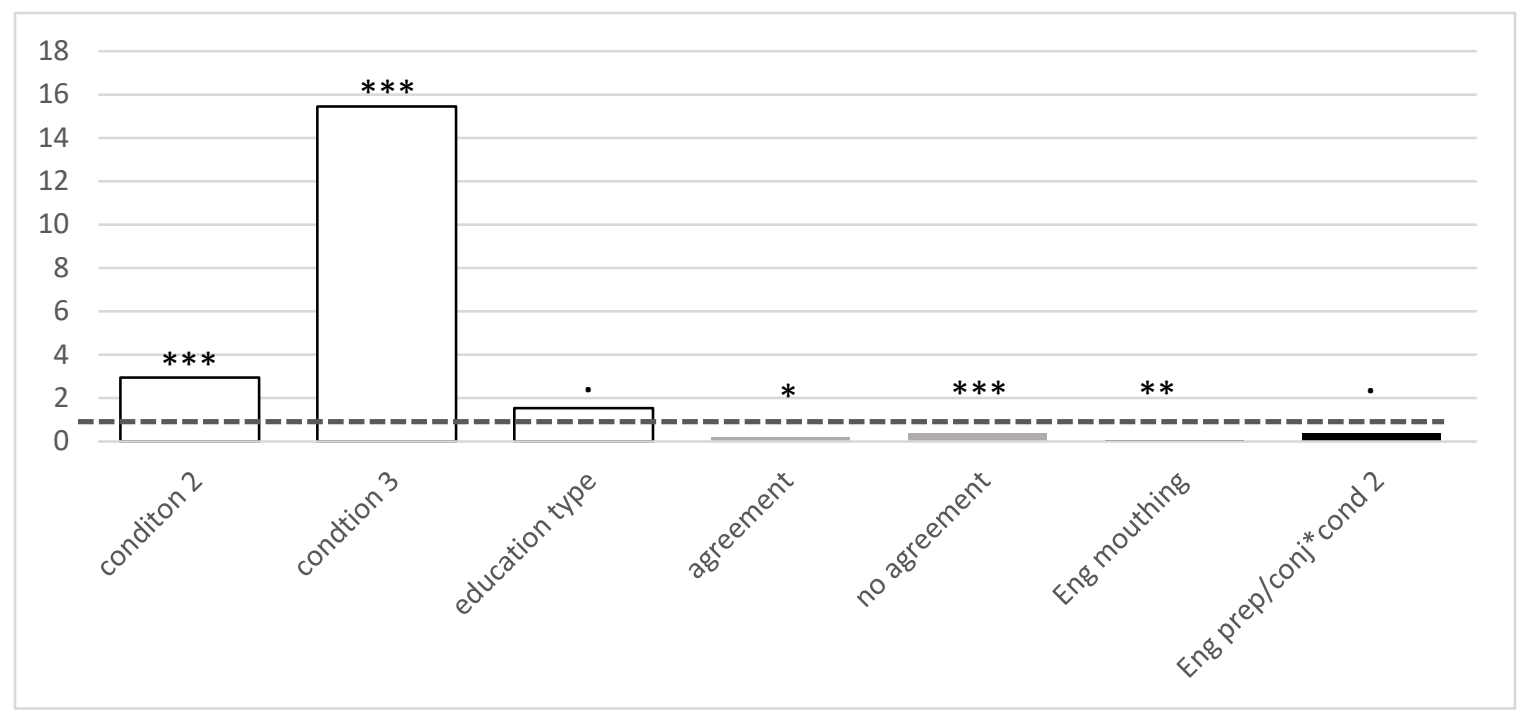

Figure 3. The odds of classifiers co-occurring with each predictor.

5.2. ClassifIERS AS OUTCOME. The second GLMM took ASL classifiers as the outcome and was also constructed using an incremental analysis. Figure 3 represents the odds calculated for each variable found to be a significant predictor of ASL classifiers. First, the odds that an average signer who was engaged in English mouthing more than the average also produced classifiers more than average (Eng mouthing, Fig. 3) are close to zero in this model with odds of 0.07, as was predicted. However, fingerspelling was found to be a highly insignificant predictor of the distribution of classifiers, so it was excluded from the model. The variable English prepositions and conjunctions' interaction with condition was also significant $(\mathrm{p}=0.05)$. The odds were only 0.38 that a signer who was producing English-based prepositions or conjunctions in condition 2 (Eng prep/conj*cond 2) more than the average was also producing classifier constructions more than average in condition 2. However, both types of ASL verbal agreement (agreeing verbs with no (overt) agreement and agreeing verbs with overt agreement, Fig. 3) were negative predictors of classifier production, and ASL indices were not a significant predictor of this distribution at all, both of which were unpredicted results. The odds that participants produced classifiers in Condition 3 were extremely high, given that this task was designed to favor the production of classifiers (odds=15.5). Among non-linguistic factors, education type

(i.e. mainstream school versus school for the Deaf) was also found to be a significant predictor of participants' classifier usage: the odds that participants who reported attending a school for the Deaf used classifier constructions more than average at any point during the study were 1.5 , as compared to those odds for participants who reported attending mainstream schools.

6. Discussion of results. The hypotheses we raised (section 4) regarding correlation among the ASL- and English-related linguistic variables were, to a significant extent, confirmed by the models described in sections 5.1 and 5.2. First, English-indexed properties tended to be positively correlated with one another, but negatively correlated with ASL-indexed properties, as expected. That is, both fingerspelling and English-based prepositions and conjunctions were significant, positive predictors of mouthing. On the other hand, classifiers and ASL indices were significant, negative predictors of mouthing. Second, English mouthing, as well as the interaction of English-based prepositions and conjunctions with condition, were strong negative predictors of classifier usage, as expected. There were some notable exceptions, however. When classifiers 
were taken as the outcome, neither ASL indices nor ASL verbal agreement, the other features we had hypothesized as being ASL-related, were significant positive predictors of classifier production; fingerspelling also did not play a significant role in that model (Fig. 3). ASL verbal agreement played no significant role (positive or negative) in the model in Figure 2. Condition and education type (mainstream school or school for the Deaf) were the only significant 'non-linguistic' predictors of the distribution of classifiers. Unlike the model in which classifiers were the outcome (Fig. 3), individual participant, age of onset of ASL acquisition, audiological status (deaf vs. hard-of-hearing), as well as the interaction between the variables classifier and participant, were all significant predictors of mouthing (Fig. 2). More prominently, hard-of-hearing participants were more likely to mouth across conditions, as compared to their deaf counterparts, which lends some independent support to the hypothesis that mouthing is an English-related feature, which would be favored by more likely exposure to English auditory input. Conversely, the fact that education type is a significant predictor of classifier usage supports this hypothesis. Participants who attended schools for the Deaf, and therefore had more opportunities to communicate manually with their peers from an early age, were more immersed in Deaf culture, and often have more positive attitudes towards ASL as a language, were more likely to produce classifiers across conditions, further supporting the idea that classifiers might index more ASL-like (or ASL-based) signing.

The fact that ASL classifiers and ASL agreeing verbs do not stand in a positivelycorrelated relationship is not entirely surprising. In many ways, the term 'classifier' is a misnomer, as nominal class is not the primary function of these items, and they behave much more like morphologically-complex verbs in ASL, unlike classifiers in some spoken languages; in fact, sign languages classifiers have also been referred to as 'polycomponental verbs' (e.g. Schembri 2003). It seems that signers who wish to express a predicate with spatial agreement have a choice between using either an ASL agreeing verb or an ASL classifier construction (both can express overt spatial agreement with their arguments). One difference between these options is that a classifier must express overt spatial agreement with its argument(s) (Sandler \& LilloMartin 2006), whereas, given our corpus, an agreeing verb may optionally drop this overt spatial agreement with its argument(s): agreeing verbs were often left plain (i.e. produced in citation form) in our data, especially among participants who reported early access to ASL.

Furthermore, although both types of predicate structures are situated at the lexical core of natural ASL according to Brentari \& Padden (2001), along with a distinct class of verbs referred to as plain verbs, this choice may be modulated by the fact that classifiers and agreeing verbs are grammatically in competition with one another, since they perform similar semantic and syntactic functions. Plain verbs obligatorily cannot express spatial agreement with their argument(s), in direct contrast to agreeing verbs and classifiers. Interestingly, for participants who produced ASL agreeing verbs with full overt spatial agreement (agreement; Fig. 3) more than the average, the odds that they also produced classifiers more than average were very low, at 0.19. However, for participants who produced ASL agreeing verbs with no overt spatial agreement (no agreement; Fig. 3), those same odds were doubled, at 0.40. Although both instantiations of spatial agreement in the uses of ASL agreeing verbs inhibited classifier production, agreeing verbs produced without spatial agreement inhibited classifiers significantly less that agreeing verbs produced with some spatial agreement.

The fact that both English mouthing and English-based prepositions and conjunctions are negative predictors of classifier usage supports the hypothesis that English-based properties 
should negatively correlate with ASL-based properties, but the fact that fingerspelling, as a linguistic property that indirectly represents English orthographic words, was not a significant predictor of the distribution of classifiers might pose a problem for this hypothesis. As Brentari \& Padden (2001) note, however, fingerspelling can be taken as an argument of classifier predicates in natural ASL, meaning those two properties can actually co-occur. Brentari \& Padden's model of the ASL lexicon is reproduced below.

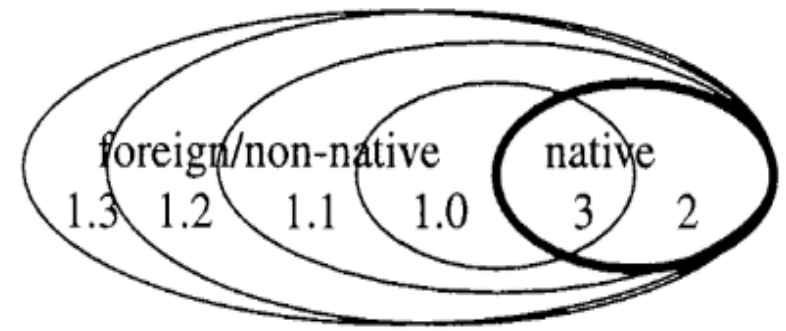

Figure 4. The ASL lexicon. (Brentari \& Padden 2001: 89)

The native parts of the lexicon, or elements of the lexicon which originate from within ASL itself, are circled in bold. Part 3 + part 1.0 represent the lexical core, and part 2 represents classifiers, or what they refer to as "polymorphemic predicates" (2001: 89). The foreign parts of the lexicon, i.e. elements that have been imported from another language (overwhelmingly English), are signs that contain one or more fingerspelled letter(s); however, as the figure makes apparent, many parts of the lexicon are overlapping, intersecting, and/or even nested inside other parts. For example, there are many initialized signs, or signs whose handshape is derived from the fingerspelled letter corresponding to the first letter of the English word, that make up some of the most common, everyday signs in natural ASL (part 1.0). Brentari \& Padden performed a battery of morphological tests in their 2001 study to determine the nesting of each part.

As a hypothesis, the fact that fingerspelling can occupy this liminal space on the periphery of the ASL lexicon (parts 1.1-1.3) could explain why it does not pattern uniformly with the more unequivocal representations of English, that is, mouthing and prepositions and conjunctions derived from the MCE systems. If classifier usage does indeed index more ASLlike signing, and fingerspelling, in some of its usages, is an integral part of the ASL lexicon, the fact that fingerspelling is not correlated (negatively or positively) with classifier constructions is not entirely surprising.

This argument might also explain why ASL indices were similarly unpredictive of the distribution of classifiers in the model represented by Figure 3. Indices, like fingerspelling, are an integral part of the ASL lexicon, so they may co-occur with classifiers just as easily as they may not co-occur with them in a given utterance. Similarly, for fingerspelling, we could say the odds that a signer who was fingerspelling was also producing a classifier predicate within that utterance are not significantly different from the odds that that participant, when s/he was producing any other type of sign, was also producing a classifier predicate within that utterance.

Furthermore, when the outcomes are reversed, and English mouthing is taken as the outcome of the model, an interesting asymmetry between the models emerges. So configured, both properties hypothesized to index English were significant, positive predictors of mouthing. This fact supports the hypothesis that fingerspelling does index English, in some of its usages, but when fingerspelling specifically is taken as a predictor of ASL classifiers specifically, the 
expected relationship does not hold, because in other instances, fingerspelling is actually a peripheral feature of the ASL lexicon. ${ }^{11}$

Finally, there are two competing sociolinguistic forces at play that might influence the type of signing participants used. On the one hand, for a variety of reasons briefly discussed in section 2, the Deaf community has limited access to natural ASL. As such, and because ASL is such an integral part of the identity of the members of this community, as a cultural group, its prestige within the community is incredibly high. At the same time, outside the community, more English-like signing is considered more prestigious. Since spoken English is the prestige language in the United States, more English-like signing indexes higher education and socioeconomic status (Lucas \& Valli 1992; Lucas 2001). As such, more English-like CSign production would be expected in more formal sociolinguistic contexts, such as an interview with an unfamiliar Deaf adult, as is the case for participants in condition 1. Looking at the results from Figure 3, in which classifiers (thought to index ASL-like signing) are the outcome, the odds that participants were producing a classifier in condition 2 were about 2.9 greater than the odds of them producing such a structure in condition 1 . In condition 3 , the odds a participant had produced a classifier were about 15.4 times greater, compared to condition 1 . This seems to indicate that participants, on average, were more likely to produce more CSign in condition 1 as compared to condition 2, and they were much less likely to produce CSign in condition 3, as compared to condition 1 . These results conform to a sociolinguistic tendency towards more English-like signing in more formal contexts: condition 1, as the most formal context, should favor the most English-like signing.

At the same time, the discourse nature of task 1, as opposed to task 3, might also explain this difference. In condition 1, participants were asked conceptual questions related to the Deaf community (the same questions asked in the interviews conducted by Lucas \& Valli 1992). This more abstract discussion might not lend itself to the spatial nature of classifier predicates, whereas condition 3, which involves the representation of many spatial relationships among agents and other entities, would have favored more spatial representations via classifier constructions. Both stories included in condition 3 were selected partially for just this reason: they showed many physical relationships among agents and other entities.

By contrast, in Figure 2, in which English mouthing is the outcome (and thought to index more English-like productions), the odds that participants were mouthing in condition 2 were about 1.6 times greater than in condition 1 . In condition 3 , the odds that participants were mouthing were only 0.6 the odds of them mouthing in condition 1 . These results support the opposite conclusion: that participants were producing more CSign in condition 2, as opposed to condition 1, and that they were producing less CSign in condition 3, as opposed to condition 1. Perhaps participants who tended to use natural ASL and were discussing topics intimately related to the Deaf community in Condition 1, were shifted towards more ASL-like signing in condition 1. On the other hand, in the highly spatial story-telling condition, participants produced more classifiers, more ASL-like signing, and less English mouthing.

6.1. IMPLICATIONS. The consensus that emerges from the background in sections 1 and 2 , is that the grammar(s) which an individual deaf learner acquired, given their exposure to ASL in the visual modality, and to English, via lip-reading, vocalization, reading and writing, may not be

\footnotetext{
${ }^{11}$ This possibility that there are two different instances of fingerspelling, one of which is clearly English-related and
} includes cases that haven't been lexicalized into ASL is also explored in Herbert \& Pires (2016). 
identical to a grammar of either natural ASL or English. This fact should not be understood in terms of deficit, meaning the child has somehow 'failed' to converge on his or her target language, as some authors in the past have claimed is for Deaf community, but a natural consequence of each individual learner's early acquisition experience. As has often been hypothesized in a different domain regarding creole linguistics, limited exposure to input from a target language, which is the case regarding natural ASL and spoken English for most of the Deaf community, can lead to innovation on the part of the child language learner (a parallel also drawn in Lucas \& Valli 1992).

The question that remains, for the purposes of this study, is: which grammar(s) underlie the production of CSign by speakers in the population modeled in this study? Is it the case that deaf signers use their knowledge of their two languages, ASL on the one hand and English on the other, in concert to produce CSign? This would entail a code-blending analysis, with separation between the grammars of ASL and English, along the lines Baker \& Van den Bogaerde (2008), who make this claim for deaf and hearing children learning Dutch Sign Language (NGT). The other possibility, as laid out in Lucas \& Valli (1992), is that CSign is governed by a third grammar, distinct from an individual's grammars of ASL and English; however, we argue, based on the extreme variability of the learning situations to which each deaf child is exposed, there are various other possibilities as well, roughly illustrated in Figure 5.

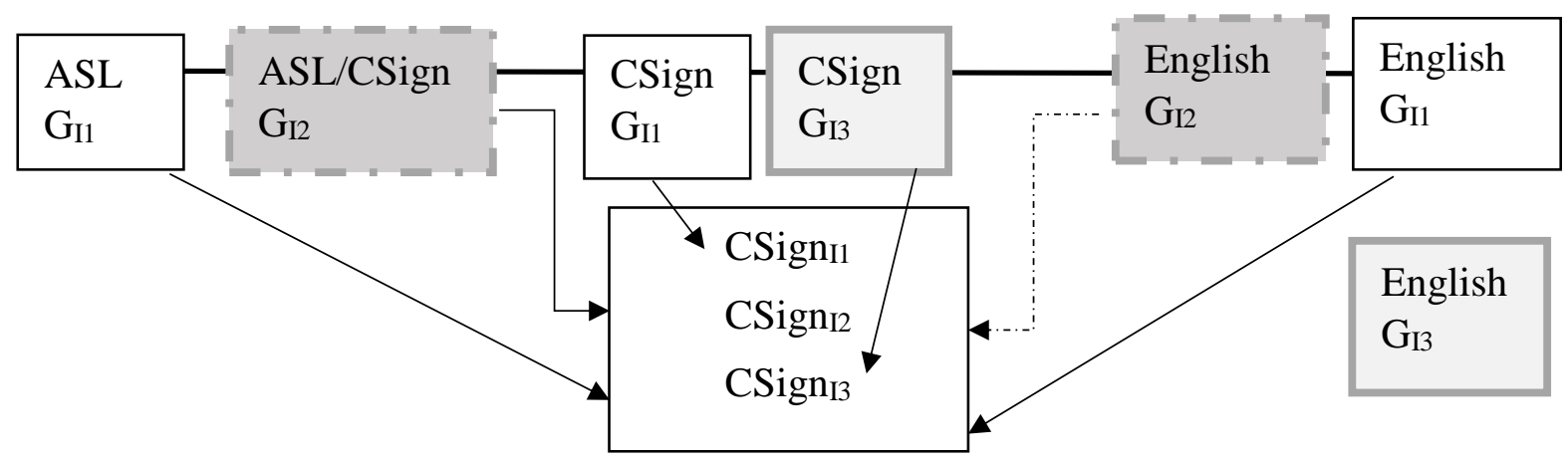

Figure 5. Hypothesized Grammars Involved in the Production of CSign.

Figure 5 illustrates the continuum that has been proposed to explain the variation in form that CSign takes, which runs from natural ASL on one extreme to spoken English on the other, with various possible grammars that might constrain its production. Let us consider three scenarios regarding the grammars of individual signers above. The first case might be individual $1\left(\mathrm{I}_{1}\right)$, who represents the classic, ideal case of a deaf child born to Deaf parents (DCDP). She has plentiful, early access to natural ASL (if her Deaf parents converged on a grammar of natural ASL), therefore she converges on a grammar that resembles natural ASL to a large extent. Then, when she goes to school, she acquires a grammar of English that is very close to the grammars onto which her typically-developing hearing peers converge. This individual might make use of her two grammars to produce $\mathrm{CSign}_{\mathrm{II}}$, although it is also possible that this individual acquired a third, separate CSign ${ }_{I 1}$ grammar, which constrains her CSign II productions, a possibility suggested by Lucas \& Valli (1992), who argue that deaf bilinguals have three distinct grammars.

The next individual, $\mathrm{I}_{2}$, might represent the more common case, also in this study, of a deaf child born to hearing parents (DCHP). This child has limited exposure to ASL in his early years, but perhaps he attended a school for the Deaf, where he started acquiring some form of 
manual communication, alongside spoken English. For this child, natural ASL may or may not be the target language, since many of the teachers and peers of $\mathrm{I}_{2}$ do not communicate in natural ASL (see Lillo-Martin 1999). This child might converge on a signed grammar that has elements of natural ASL and CSign, represented by ASL/CSign $\mathrm{G}_{12}$. I 2 may or may not converge on a 'native-like' grammar of spoken English, given his delayed language acquisition and the physiological barrier that constrains his access to that language. Therefore, his CSign I2 production might be constrained by two grammars, ASL/CSign $\mathrm{G}_{12}$ and English $\mathrm{G}_{12}$, which may have different properties from the corresponding grammars of $\mathrm{I}_{1}$.

$\mathrm{I}_{3}$, with $\mathrm{CSign}_{\mathrm{I}}$ and English $_{\mathrm{I}}$ grammars, may be an individual with relatively heavy spoken English input and late exposure to ASL. This situation might be representative of hardof-hearing children, children who are born hearing but become deaf early in life, or deaf children who have a successful cochlear implant surgery. I $_{3}$ most likely had delayed and restricted access to manual input, perhaps even acquiring that language well into adulthood. Figure 5 represents the possibility that the CSign production by $\mathrm{I}_{3}$ results from a single grammar, $\mathrm{CSign} \mathrm{G}_{3}$, that may also be heavily influenced by English, although she has also a parallel English grammar, English $\mathrm{G}_{3}$, that may not necessarily underlie her CSign production directly.

The scenarios described above correspond to two approaches to the grammatical knowledge underlying CSign production. In one approach, the code-blending approach, at least two grammars (one with more features from ASL and the other with more features from English) underlie CSign production. This was illustrated considering the case of individuals $I_{1}$ and $I_{2}$, although the approach could then be extended to other individuals, such as $\mathrm{I}_{3}$, with the only difference that the set of grammars involved would vary for each individual. The second approach, the single-grammar approach, CSign production would be determined by a single grammar, as we illustrated only for individual $\mathrm{I}_{3}$. This option, that some deaf bilinguals converge on a Csign manual grammar that uniquely determines all their Csign production, is more difficult to defend, for various reasons we discuss below.

Mufwene's (2008:115) analysis of language as a diverse collection of idiolects, or individual I-grammars, specific to each individual speaker in a given speech community, is also compatible with the two-grammar approach. Under this view, speakers (signers here) necessarily maintain more than one grammar variant. Individual learners usually maintain one variant that is dominant in their particular social network, such as their school for the Deaf or their Deaf peer network, and one or more variants that are (partially) accessible in cases when the individual learns and uses aspects of the variants of other speakers.

We take the code-blending approach, with at least two distinct grammars underlying CSign, as more compatible with the results of the current study, such as the ones listed below.

a. English-based properties tended to negatively predict ASL-based properties and positively predict other English-properties.

b. ASL-based properties generally were negative predictors of English-based properties

c. Hard-of-hearing participants more likely to mouth across conditions as compared to their severely to profoundly deaf counterparts

d. Asymmetry in classifier usage by education type

e. Participant, classifier* participant were significant predictors of English mouthing

Table 2: Evidence for a code-blending analysis of CSign 
First, the general trend that English-based properties tended to positively predict other English-based properties, while they negatively predicted ASL-based properties, supports the general framework proposed in this paper, that CSign is determined by at least two different grammars, running from natural ASL and more ASL-like grammars on one extreme to more English-like grammars on the other. This approach is also supported by the fact that ASL-like properties tended to negatively predict English-based properties in the results we presented. These and the other results reported provide evidence for the separate contribution of two distinct grammars (one more ASL-like and the other one more English-like) underlying the CSign production of different signers.

The remaining results (c-d) listed in Table 2 show clear evidence of distinction among signers that would produce English-like properties (mouthing) or ASL-like properties (classifiers) in their production, although they all still showed evidence of substantial variation in their production. This includes the fact that education type, mainstream or school for the Deaf, is predictive of classifier usage, which can be explained if signers with different language learning trajectories show different levels of access to ASL-like features in the underlying grammars. Regarding mouthing, our results also indicate that it is a prominent property of CSign. If the assumption that previous literature has made about English mouthing being relatively infrequent in natural ASL data holds, then the high frequency of mouthing found in the data from this study (accompanying $~ 74 \%$ of all signs) is strong evidence for this hypothesis. However, if mouthing were simply lexicalized to a number of natural ASL signs in CSign production, we might not expect to see a significant trend across participants in terms of their mouthing production. It is then highly telling that hard-of-hearing participants, who had a greater amount of auditory exposure to English, mouthed more often across conditions than their severely to profoundly deaf counterparts. Finally, result (e) in Table 2 provides broad evidence of variable I-grammars underlying the production of CSign, since participant, and the interaction between participant and classifier usage (meaning some participants produce classifiers more than others) was a significant predictor of (variation in) English mouthing. This is to say the degree to which each participant produces mouthing, and classifiers, varies across participants.

In sum, the results reported in this paper provide evidence supporting an approach in which CSign across different deaf and hard-of-hearing individuals is determined by at least two distinct underlying I-grammars, yielding sign production (with substantial co-occurrence of mouthing) that we analyze as representing a type of code-blending, in which both ASL-like and English-like features can be identified. ${ }^{12}$

7. Conclusion and Future Research. The results of this study clearly indicate that there are individual differences among the productions of individual deaf signers, and that these differences should not be understood in terms of some sort of deficit on the part of the deaf language learners, as some previous research has suggested, but rather a natural consequence of highly variable language acquisition situations across deaf individuals. This conclusion makes the very notion of 'CSign in the American Deaf Community' more complex: if each individual's CSign production is slightly different from the next individual's, then the notion of CSign as a monolithic whole, even a variable one, is difficult to conceptualize. Taken together, the results of this study point towards the idea that many individual deaf ASL-English bilinguals have at least

\footnotetext{
12 It remains to be determined more precisely whether at least some of these individuals show evidence of having a third, mixed-grammar that would also underlie part of their CSign production, as suggested by Lucas \& Valli 1992.
} 
two distinct mental grammars, one that is closer to English, and one that is more like natural ASL, which together constrain their CSign productions. In addition, we analyze CSign production as a type of bimodal code-blending, in which ASL-like and English-like grammatical features underlie different aspects of CSign production.

\section{References}

Baker, Anne and Beppie Van den Bogaerde. 2008. Code-mixing in signs and words in input to and output from children. Sign Bilingualism: Language development, interaction, and maintenance in sign language contact situations. 1-28.

Berent, Gerald P. 2004. Sign Language-Spoken Language Bilingualism: Code Mixing and Mode Mixing by ASLEnglish Bilinguals. The handbook of bilingualism. 312-335.

Brentari, Diane, and Carol Padden. 2001. Native and foreign vocabulary in American Sign Language: A lexicon with multiple origins. Foreign vocabulary in sign languages. 87-120.

Chomsky, Noam. 2005. Three factors in language design. Linguistic inquiry 36(1). 1-22.

Cokely, Dennis. 1983. When is a Pidgin Not a Pidgin?: An Alternate Analysis of the ASL-English Contact Situation. Sign Language Studies 38(1). 1-24.

Davis, Jeffrey. 1989. Distinguishing language contact phenomena in ASL interpretation. The sociolinguistics of the deaf community. 85-102.

Emmorey, Karen, Helsa Borinstein, and Robin Thompson. 2005. Bimodal Bilingualism: Code-blending between Spoken English and American Sign Language. ISB4:Proceedings of the $4^{\text {th }}$ International Symposium on Bilingualism. 663-673.

Herbert, Marjorie and Acrisio Pires. 2016. Mouthing, fingerspelling, or both? Codeswitching and Lexical Borrowing in American Sign Language (ASL). Paper presented at UIC Bilingualism Forum.

Lee, Dorothy M. 1982. Are There Really Signs of Diglossia?: Re-Examining the Situation. Sign Language Studies 35(1). 127-152.

Liddell, Scott. 2000. Indicating verbs and pronouns: Pointing away from agreement. The signs of language revisited: An anthology to honor Ursula Bellugi and Edward Klima. 303-320

Lillo-Martin, Diane. 1999. Modality effects and modularity in language acquisition: The acquisition of American Sign Language. Handbook of child language acquisition. 531- 567.

Lucas, Ceil. 2001. The sociolinguistics of sign languages. Cambridge University Press.

Lucas, Ceil, and Clayton Valli. 1989. Language Contact in the American Deaf Community. The sociolinguistics of the Deaf community. 11-40.

-- $\quad$ 1992. Language Contact in the American Deaf Community.

Mufwene, Salikoko. 2008. Competition and selection in language evolution. Language Evolution. 115-131.

Sandler, Wendy and Diane Lillo-Martin. 2006. Sign Language and Linguistic Universals.

Schembri, Adam. 2003. Rethinking "classifiers" in signed languages. Perspectives on classifier constructions in sign languages. 3-34.

Stokoe Jr, William C. 1969. Sign Language Diglossia. 